\title{
TWO COLOUR CCD PHOTOMETRY OF MALIN-CARTER SHELL GALAXIES
}

\author{
A. Wilkinson \\ Astronomy Dept., The University, Manchester M13 9PL, England. \\ W.B. Sparks \\ RGO, Herstmonceux Castle, East Sussex BN27 1RP, England. \\ D. Carter \\ Mount Stromlo Observatory, Canberra ACT 2606, Australia. \\ D.A. Malin \\ AAO, P.O. Box 296, Epping NSW 2121, Australia.
}

\section{INTRODUCTION}

Shells may be the result of the disruption of a small companion in the potential of a much larger galaxy (e.g., Hernquist \& Quinn, 1986; Dupraz \& Combes, 1985), the disturbance of a disk system in a tidal encounter (Toomre, 1972), an accumulation of resonant stellar orbits (Binney, pers.comm.) or the result of some shock phenomenon in a hot galactic atmosphere (e.g., Williams \& Christiansen, 1985; Wilkinson \& Bailey, 1985;). To distinguish between these formation mechanisms, CCD direct images in B and $\mathrm{R}$ have been obtained at the Anglo-Australian $3.8 \mathrm{~m}$ telescope for 66 of the 74 galaxies in the range $01^{h} 40^{\prime}<\alpha<13^{h} 46^{\prime}$ in the MalinCarter (1983) catalogue of shell galaxies.

\section{IMAGE PROCESSING}

Figs. 1a) to d) show different ways of presenting the same image.

a)

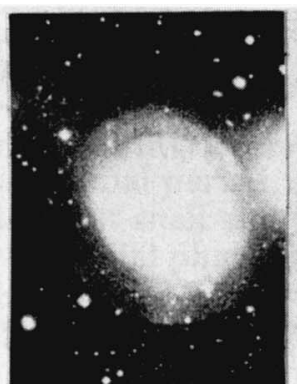

b)

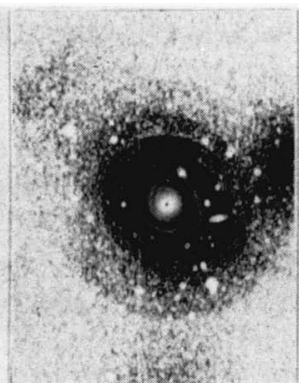

c)

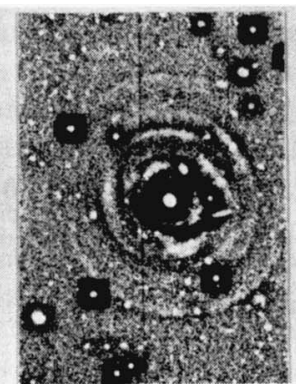

d)

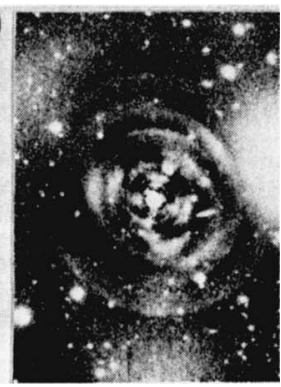

Figure 1. 0422-476 a) Direct CCD image after routine processing. b) Logarithmic gradient. Bright where surface brightness profile is steep, dark areas have shallower profile. c) Unsharp masking. Smoothed image subtracted from normal image. d) Modelling and subtraction of primary galaxy elliptical surface brightness profile. 


\section{RESULTS}

Our observations suggest three structure categories. a) Cone - Shells aligned with galaxy major axis, interleaved in radius on alternate sides of galaxy. (Fig. 2a). b) Randomly distributed arcs - Shells sharper-edged than in (a), irregularly distributed all around rather circular galaxy (Fig. 1a). c) Irregular - Shells distributed irregularly, not clearly related to any projected principal axis (Fig. 2b).

a)

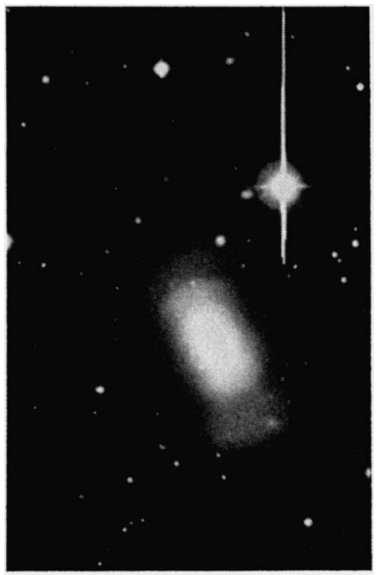

b)

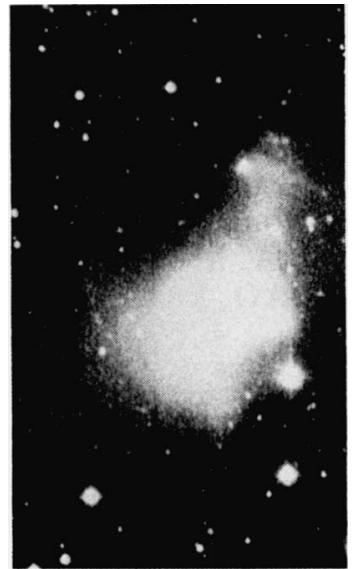

Figure 2. a) 0315-550, direct image, aligned shells b) 0515-541, direct image, irregular shells.

1) The gradient maps show that shells commonly occur close to the nucleus. In roughly $20 \%$ of the systems these innermost shells (Fig. 1b) have spiral morphology. The galaxy light profiles are quite well represented by an $r^{1 / 4}$ law, so any inner disk does not dominate the light profile.

2) Despite the very disturbed appearance of the irregular system 1208-337 the shells still interleave, with the ratio of shell radii from one side to the other being almost constant at $\simeq 1.2$.

3) 0422-476 is a member of the 'randomly distributed arc' category. The outer shell here has the same radius all around the galaxy, although with occasional breaks (Fig. 1a). An ellipse fitted to the upper and lower arcs has a major axis $\simeq 4.3 \mathrm{arcmin}$ but its centre lies within 2 arcsec of the nucleus.

4) The galaxies have an enormous diversity of central surface brightness. This suggests that a wide range of potentials allow shells to develop.

\section{REFERENCES}

Dupraz, Ch. \& Combes, F., 1986. Astron. Astrophys., preprint.

Hernquist, L. \& Quinn, P.J., 1986. Astrophys. J., preprint.

Malin, D.F. \& Carter, D., 1983. Astrophys. J., 274, 534.

Toomre,A. \& Toomre, J., 1972. Astrophys. J., 178, 623.

Wilkinson, A. \& Bailey, M.E., 1985. Cosmical Gas Dynamics, ed. F.D. Kahn., p 63.

Williams, R.E. \& Christiansen, W.A.,1985. Astrophys. J., 291, 80. 